\title{
QGSJET-II: physics, recent improvements, and results for air showers
}

\author{
S. Ostapchenko ${ }^{1,2, a}$ \\ ${ }^{1}$ NTNU, Institutt for fysikk, 7491 Trondheim, Norway \\ ${ }^{2}$ D.V. Skobeltsyn Institute of Nuclear Physics, Moscow State University, 119992 Moscow, Russia
}

\begin{abstract}
Modeling of high energy hadronic and nuclear interactions by the QGSJET-II generator is discussed. Recent updates related to the treatment of nonlinear effects in the interaction dynamics and to the model calibration with new LHC data are described. A special attention is devoted to the predictions of the new model version for characteristics of extensive air showers initiated by high energy cosmic rays. In particular, an improved description of charge exchange processes in pion collisions is discussed and the respective enhancement of the shower muon content is analyzed.
\end{abstract}

\section{Introduction}

Despite the long history of high energy cosmic ray (CR) studies, there remain numerous unsolved problems in the field. Among the most outstanding ones is the determination of the primary CR composition at ultra high energies. Apart from purely experimental challenges, a serious uncertainty in the interpretation of data is related to the description of nuclear-electromagnetic cascades in the atmosphere - extensive air showers (EAS) - which are the main tool for detecting CR primaries in very high energy range. The largest part of that uncertainty stems from the treatment of hadron-air interactions in the shower by corresponding models since the latter have to be extrapolated from much lower accelerator energies. Therefore, the start of the Large Hadron Collider (LHC) and the success of its experimental program are of great importance for the CR field. There is a strong hope that new data will substantially constrain EAS simulation procedures and contribute to a successful resolution of ultra-high energy cosmic ray (UHECR) puzzles.

In the following I discuss recent updates of the QGSJET-II hadronic interaction model [1-3], both concerning improvements in the underlying theoretical framework and regarding its re-calibration with new LHC data. A special emphasis will be given to how those modifications impact the predicted EAS characteristics.

\section{Overview of the model}

\subsection{Semihard processes}

Already 30 years ago one realized the importance of socalled semihard processes, which give rise to production of hadron jets of relatively large transverse momenta $p_{t}$, for high energy interactions of hadrons [4]. Apart from a

\footnotetext{
ae-mail: sergey.ostapchenko@ntnu.no
}

quick energy rise of the respective contributions and their strong impact on characteristics of hadronic collisions, notably, on the multiplicity of produced secondaries, there was a hope to develop a fully perturbative treatment for a general minimum bias hadron scattering - since the underlying physics involves parton cascades characterized by large transferred momentum.

In an attempt to include such processes into modelsgenerators of hadronic collisions a so-called minijet approach has been proposed (e.g. [5, 6]). The latter was based on a rather radical assumption, namely, that properties of purely "soft" (small momentum transfer) processes do not depend on energy and all the non-trivial physics, like the energy-rise of total and inelastic cross sections, multiplicity, etc. is due to a quickly rising minijet contribution, the latter corresponding to high $p_{t}^{2}>Q_{0}^{2}$ processes, $Q_{0}$ being some characteristic momentum scale. In early 90th, there have been extensive discussions on the "true" value of the respective $Q_{0}$-cutoff which proved to be a crucial parameter for the scheme. Moreover, one speculated that this cutoff should be energy-dependent (e.g. [7]) in order to account for parton saturation effects $[4,8]$ and should depend on nuclear mass numbers when treating nuclear collisions [9].

The motivating idea behind the original QGSJET model [10] was that there is no principal difference between soft and hard processes in hadron scattering. Correspondingly, the $Q_{0}$-cutoff is just a technical parameter: it merely separates the kinematic region which we attempt to describe by perturbative methods from long-distance physics for which we are forced to apply a phenomenological description. In order to develop a unified scheme, both soft and semihard parton cascades have been treated in QGSJET and, later, in the NEXUS model [11] in the framework of Gribov's Reggeon Field Theory (RFT) [12], describing them as exchanges of "soft" and "semihard" Pomerons, as discussed in more detail in [13]. The soft 
Pomeron is used as an effective description for a nonperturbative cascade characterized by small parton virtualities, $q^{2} \simeq p_{t}^{2}<Q_{0}^{2}$. As semihard processes involve parton cascades which develop, at least partly, in the region of high virtualities, $q^{2}>Q_{0}^{2}$, the respective part of parton evolution can be treated using perturbative methods. Correspondingly, the "semihard Pomeron" is defined as a $t$-channel iteration of the soft one and of a piece of parton ladder built by means of DGLAP evolution - Fig. 1. Thus,

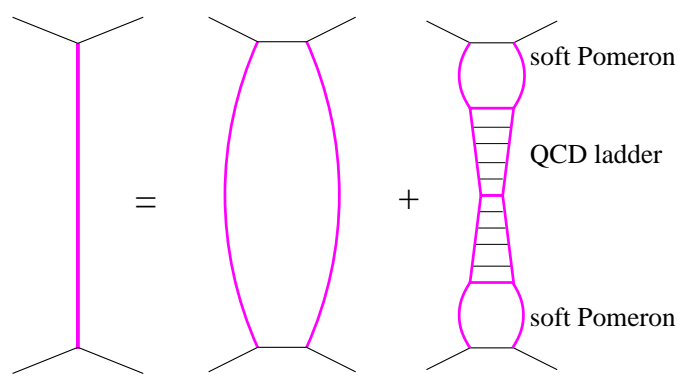

Figure 1. "General Pomeron" is the sum of the soft and semihard ones - respectively the first and the second graphs in the r.h.s.

a general "elementary" scattering contribution is described by a "general Pomeron", i.e. by a sum of the soft and semihard ones, as shown in the Figure.

\subsection{Multiple scattering}

A general hadron-hadron (hadron-nucleus, nucleusnucleus) scattering involves multiple scattering processes, i.e. multi-Pomeron exchanges, as shown in Fig. 2. The

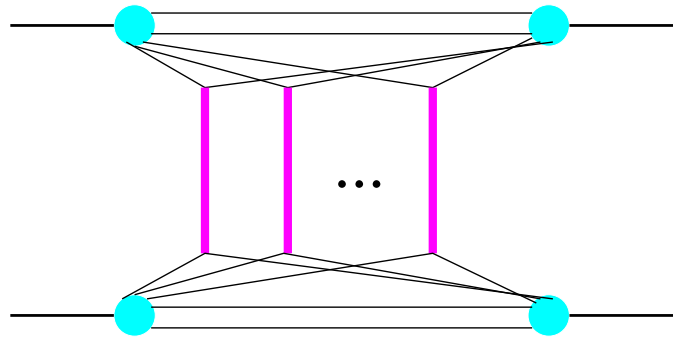

Figure 2. General multi-Pomeron contribution to hadron-hadron scattering amplitude; elementary scattering processes (vertical thick lines) are described as Pomeron exchanges.

beauty of the RFT formulation is that it allows one to develop a coherent approach for calculating various interaction cross sections and for treating particle production. While the total cross section is related via the optical theorem to the imaginary part of elastic scattering amplitude, the latter being defined by the summary contribution of the graphs of the kind depicted in Fig. 2, various partial cross sections may be derived from unitarity cuts of those graphs, obtained with the help of so-called AbramovskiiGribov-Kancheli (AGK) cutting rules [14]. The physical process behind a particular cut diagram may be easily understood if one associates a cut Pomeron with a real parton cascade which gives rise to secondary hadron production, while an uncut one corresponds to a virtual (elastic) rescattering process. Hence, for any partial contribution, e.g. for the one with precisely two elementary particle production contributions (two cut Pomerons), one has to sum up contributions of graphs with arbitrary numbers of virtual re-scatterings (uncut Pomerons).

\subsection{Nonlinear effects}

The crucial development implemented in QGSJET-II is the treatment of nonlinear interaction effects related to merging (splitting) of parton cascades, which are described in the model by so-called enhanced (Pomeron-Pomeron interaction) diagrams $[15,16]$. As before, various partial cross sections are then obtained considering relevant unitarity cuts of elastic scattering graphs, applying the AGK cutting rules $[17,18]$. The corresponding calculation procedure forms the basis for generating arbitrary (generally complicated) configurations of hadronic and nuclear collisions in a Monte Carlo fashion [3], thus providing a microscopic treatment for the interaction dynamics.

An important example is the description of diffraction dissociation processes in hadronic and nuclear collisions, in which multi-particle production patterns are characterized by a formation of large rapidity gaps (LRG) not covered by secondary hadrons [18]. While almost all the other models-generators employ an ad-hoc treatment for the diffraction, e.g. using some parametrized ansatz for the diffractive mass $M_{X}$ distribution, QGSJET-II predicts the relevant characteristics, based on the structure of the underlying RFT diagrams. As an illustration, QGSJETII-04 [3] prediction for $\xi$-distribution $\left(\xi=M_{X}^{2} / s, s\right.$ being the c.m. energy squared for the collision) for single diffractive proton-proton collisions at $\sqrt{s}=7 \mathrm{TeV}$ is compared to the one of SYBYLL 2.1 [19] in Fig. 3. While

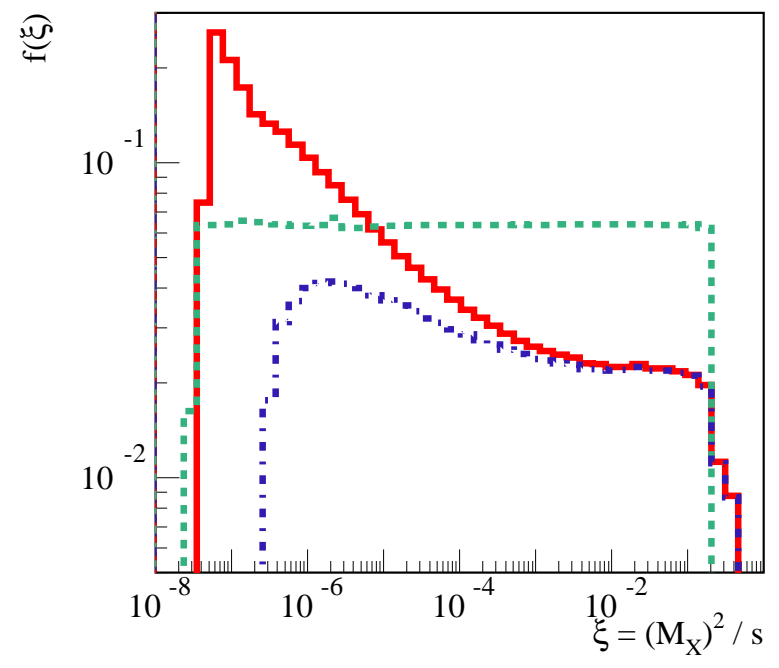

Figure 3. $f_{\mathrm{SD}}(\xi) \equiv \frac{\xi}{\sigma_{\mathrm{SD}}} \frac{d \sigma_{\mathrm{SD}}}{d \xi}$ for single diffractive $p p$ collisions at $\sqrt{s}=7 \mathrm{TeV}$ as obtained using QGSJET-II-04 (solid) and SIBYLL 2.1 (dashed), also shown is the contribution to $f_{\mathrm{SD}}(\xi)$ from high mass diffraction in QGSJET-II-04 (dash-dotted).

in SYBYLL 2.1 the assumed $d M_{X}^{2} / M_{X}^{2}$ distribution produces the flat $\xi$-shape, a more complicated $M_{X}^{2}$-distribution 
is obtained in the case of QGSJET-II-04: apart from a sharp peak at low $\xi$, formed by the contribution of low mass diffraction (small $M_{X}^{2}$ ), one obtains also a nontrivial $M_{X}^{2}$-dependence for the partial contribution of high mass diffraction, which is related to the different strength of nonlinear screening corrections in peripheral (large impact parameter $b$ ) and central (small $b$ ) $p p$-collisions $[18,20]$. Such kind of $M_{X}^{2}$-dependence is indeed indicated by the ATLAS LRG data [21] though the decisive discrimination may be performed by the TOTEM experiment [22].

The developed scheme is based on the assumption that Pomeron-Pomeron coupling corresponds to merging of parton cascades in the low virtuality domain, $q^{2}<Q_{0}^{2}$, being dominated by soft processes. The strength of nonlinear interaction effects thus depends on the so-called triplePomeron coupling $G_{3 \mathbb{P}}$ which is the main parameter of the approach. It is worth stressing that a consistent treatment requires a full resummation of enhanced diagrams, to all orders with respect to $G_{3 \mathbb{P}}$, both for uncut (elastic scattering) diagrams and for various unitarity cuts, which has been performed in Refs. [16-18]. As an illustration, shown in Fig. 4 is the sequence of cut enhanced graphs corre-

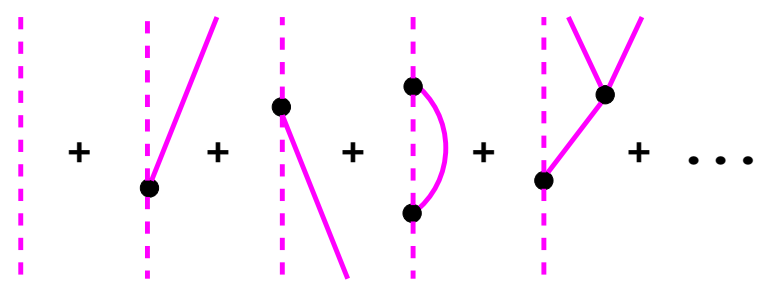

Figure 4. Cut enhanced graphs corresponding to single "elementary" particle production process. Cut Pomeron shown by a thick dashed line represents a real parton cascade which gives rise to secondary hadron production. Uncut Pomerons (corresponding to virtual parton cascades) are shown by thick solid lines and describe elastic re-scattering of the (real) cascade partons off each other and off the projectile and target hadrons (nuclei).

sponding to a single real parton cascade (cut Pomeron), with an arbitrary number of elastic re-scatterings of the partons off each other and off the projectile and target hadrons (nuclei); the process gives rise to a production of a single chain of secondary hadrons stretched between the projectile and the target, without any LRG.

While the previous model version, QGSJET-II-03 [23], was based on resummation of (dominant) enhanced graphs of "net"-like type, also so-called Pomeron "loop" graphs 1 have been taken into consideration in QGSJET-II-04 [3], i.e. the new treatment takes into account all significant diagrams for Pomeron-Pomeron interactions.

In contrast to the minjet approach, the above-discussed treatment allows one to obtain a consistent description of the observed energy-dependence of total and elastic proton-proton cross sections for a fixed energyindependent $Q_{0}$-cutoff between soft and hard processes,

\footnotetext{
${ }^{1}$ Pomeron loop graphs contain multi-Pomeron vertices connected to each other by two or more Pomeron sequences $[17,18]$. Though being formally sub-leading, those diagrams provide important contributions to elastic scattering amplitude, in particular, at large $b$.
}

while staying in agreement with measured proton structure functions $[1,3]$. To understand that, let us examine the standard ansatz of the minijet approach for the contribution of semihard processes to the interaction eikonal $\chi_{\mathrm{sh}}(s, b)$, as implemented in almost all the Monte Carlo generators on the market. Namely, one assumes $\chi_{\text {sh }}$ to be given by the product of the inclusive minijet production cross section for $p_{t}^{2}>Q_{0}^{2}, \sigma_{\text {jet }}\left(s, Q_{0}^{2}\right)$, and the overlap function $A(s, b)$, the latter being the convolution of the projectile and target proton form factors for a given impact parameter $b$ [6]. This contains a strong (and unjustified) assumption that hard processes contribute to non-incusive quantities like $\chi_{\mathrm{sh}}(s, b)$ in the same way as they do for inclusive jet spectra: $\sigma_{\text {jet }}$ is defined by a convolution of a parton scatter cross section $d \sigma_{I J}^{2 \rightarrow 2} / d p_{t}^{2}$ with the universal parton distribution functions (PDFs) of protons $f_{I}\left(x, q^{2}\right)$ :

$$
\begin{aligned}
\sigma_{\text {jet }}\left(s, Q_{0}^{2}\right)=\int d x_{1} d x_{2} \int d p_{t}^{2} \sum_{I, J=q, \bar{q}, g} \frac{d \sigma_{I J}^{2 \rightarrow 2}}{d p_{t}^{2}} \\
\times f_{I}\left(x_{1}, p_{t}^{2}\right) f_{J}\left(x_{2}, p_{t}^{2}\right) .
\end{aligned}
$$

Using this ansatz, nonlinear screening corrections may only emerge in the evolution of the universal PDFs, corresponding to a re-scattering of intermediate partons off the parent hadron. Hence, a strong damping of the low $x$ rise of $f_{I}\left(x, q^{2}\right)$ is required in order to stay in agreement with observed energy-dependence of $\sigma_{p p}^{\text {tot }}$. In contrast, in the more general treatment based on enhanced diagrams, $\chi_{\mathrm{sh}}(s, b)$ is expressed via non-universal PDFs which contain additional screening corrections due to intermediate parton re-scattering off the partner hadron [1], as depicted in Fig. 5. It is this additional screening contribution which
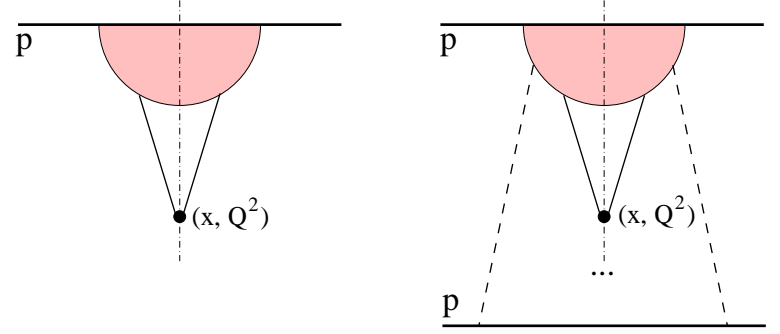

Figure 5. Schematic view of parton distributions as "seen" in deep inelastic scattering (DIS) - left, and in proton-proton collisions - right. Low $x$ parton (sea quark or gluon) originates from the initial state "blob" and interacts with a virtual "probe". The universal PDFs measured in DIS are affected by a re-scattering of intermediate partons from the initial state cascade (hidden in the "blob") off the parent proton. In proton-proton interactions the initial "blob" is affected itself by the collision process: due to a re-scattering of intermediate partons off the partner (here, target) proton, as indicated by dashed lines in the Figure.

damps the energy-rise of hadronic cross sections without affecting the low $x$ behavior of the universal PDFs. It is noteworthy that the above-discussed effect may be interpreted alternatively as being due to correlations in generalized multi-parton distributions [24].

Finally, it is important to stress that the abovediscussed treatment remains consistent with pQCD in the 
two key aspects: the evolution of universal PDFs is still governed by DGLAP equations and the collinear QCD factorization does apply to inclusive jet spectra (e.g. Eq. (1) remains valid), as discussed in more detail in [1].

\subsection{Main drawbacks of the model}

One of the drawbacks of the present treatment is related to the underlying assumption that Pomeron-Pomeron coupling is dominated by "soft" parton processes at $q^{2}<Q_{0}^{2}$. Correspondingly, the treatment doesn't take into account "hard" Pomeron-Pomeron coupling corresponding to nonlinear parton processes in the perturbative domain, thus missing the respective screening effects. This poses limitations to model applications for a treatment of central nucleus-nucleus collisions at very high energies.

Another serious drawback which is common to most of the models-generators on the market is related to using eikonal Pomeron-hadron and multi-Pomeron vertices, i.e. the treatment neglects energy-momentum sharing between multiple scattering processes at the amplitude level [25] (see also a qualitative discussion in [26]). A construction of a model which combines a treatment of the energysharing mechanism with the one for nonlinear interaction effects remains a serious challenge at present.

\section{Air shower characteristics}

\subsection{Impact of LHC data on the model predictions for $X_{\max }$ and $\mathrm{RMS}\left(X_{\max }\right)$}

The impact of the main improvement implemented in QGSJET-II-04, the treatment of Pomeron loop contributions, on EAS characteristics has been investigated previously using a preliminary version of the model [27]. It appeared that the differences with the respective QGSJET-II03 predictions were quite small, which may be surprising, given the relatively large contributions of Pomeron loop graphs [18]. The reason is that in the complete scheme one has to use a substantially smaller (almost by a factor of two) value for the triple-Pomeron coupling $G_{3 \mathbb{P}}$ in order to stay in agreement with collider measurements of total and elastic proton-proton cross sections. Thus, the scheme realized in QGSJET-II-03, which was based of "net"-like enhanced Pomeron graphs only but used a larger $G_{3 \mathbb{P}}$ value, turned out to be a reasonable approximation to the complete treatment, the latter including also Pomeron loop diagrams and using smaller $G_{3 \mathbb{P}}$.

Additional improvement in the final model version has been its calibration with LHC data, notably, with TOTEM results on $\sigma_{p p}^{\text {tot/el }}$ [28]. This resulted e.g. in a somewhat slower energy rise of hadronic cross sections, as illustrated in Fig. 6 and discussed in more detail in [29]. In turn, this leads to a slightly higher elongation rate for the shower maximum position, with the difference to QGSJET-II-03 for the predicted $X_{\max }$ reaching $20 \mathrm{~g} / \mathrm{cm}^{2}$ at the highest CR energies - Fig. 7(left). Similarly, the smaller $\sigma_{p \text {-air }}^{\text {inel leads }}$ to larger fluctuations of $X_{\max }$, the corresponding standard deviation $\operatorname{RMS}\left(X_{\max }\right)$ being now in a good agreement with the old QGSJET results - Fig. 7(right).

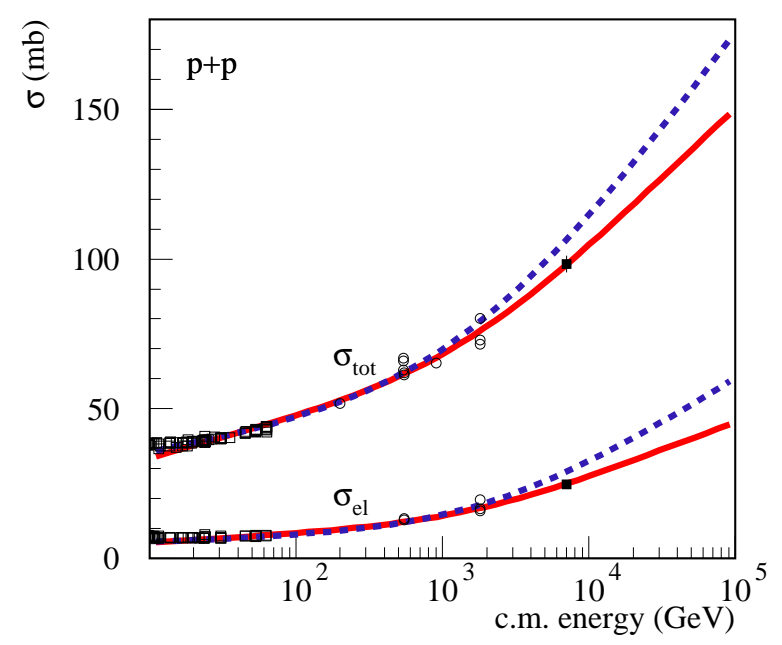

Figure 6. Total and elastic proton-proton cross sections as calculated using QGSJET-II-04 (solid) and QGSJET-II-03 (dashed) compared to accelerator data (points); solid squares - TOTEM results [28], open symbols - compilation of data from Ref. [30].

The latter circumstance is not occasional but reflects the fact that model predictions for EAS fluctuations are now strongly constrained by the TOTEM results for proton-proton cross sections. ${ }^{2}$ In turn, fluctuations of air showers induced by relatively heavy nuclei depend mostly on the geometry of nucleus-nucleus interactions, notably on the variations of the impact parameter for a collision and the related fluctuations of the number of "wounded" projectile nucleons participating in secondary particle production, and on the fragmentation of the nuclear spectator part [31-33]. As the consequence, model predictions for $\operatorname{RMS}\left(X_{\max }\right)$ for iron-induced EAS coincided rather well even in the pre-LHC era. However, there is still an uncertainty concerning the average $X_{\max }$, as illustrated e.g. by the difference between the respective QGSJET and QGSJET-II-04 predictions, which reflects the uncertainty in the energy dependence of the inelasticity of hadron-air collisions.

\subsection{Treatment of charge exchange in pion collisions and EAS muon content}

Let us now turn to the muon content of extensive air showers, for which one has a persistent disagreement between the results of the Pierre Auger Observatory (PAO) and the respective predictions from EAS simulations [34]. The standard option for increasing the muon number $N_{\mu}$, a much faster energy rise of the multiplicity $N_{\text {ch }}$ of charged particles produced, now seems to be closed by LHC data. Indeed, the relevant experimental results appear to be well-bracketed by the predictions of interaction models used in EAS simulations [35]. Another potential option for increasing $N_{\mu}$, a copious production of (anti-)baryons $[36,37]$, is neither supported by LHC data. ${ }^{3}$

\footnotetext{
${ }^{2}$ QGSJET prediction for $\sigma_{p p}^{\text {inel }}$ appeared to be in a good agreement with the TOTEM measurements.

${ }^{3}$ Moreover, an enhancement of the number of high energy muons $\left(E_{\mu} \gtrsim 10\right) \mathrm{GeV}$ seems to be suggested by the PAO data, while the "baryon
} 

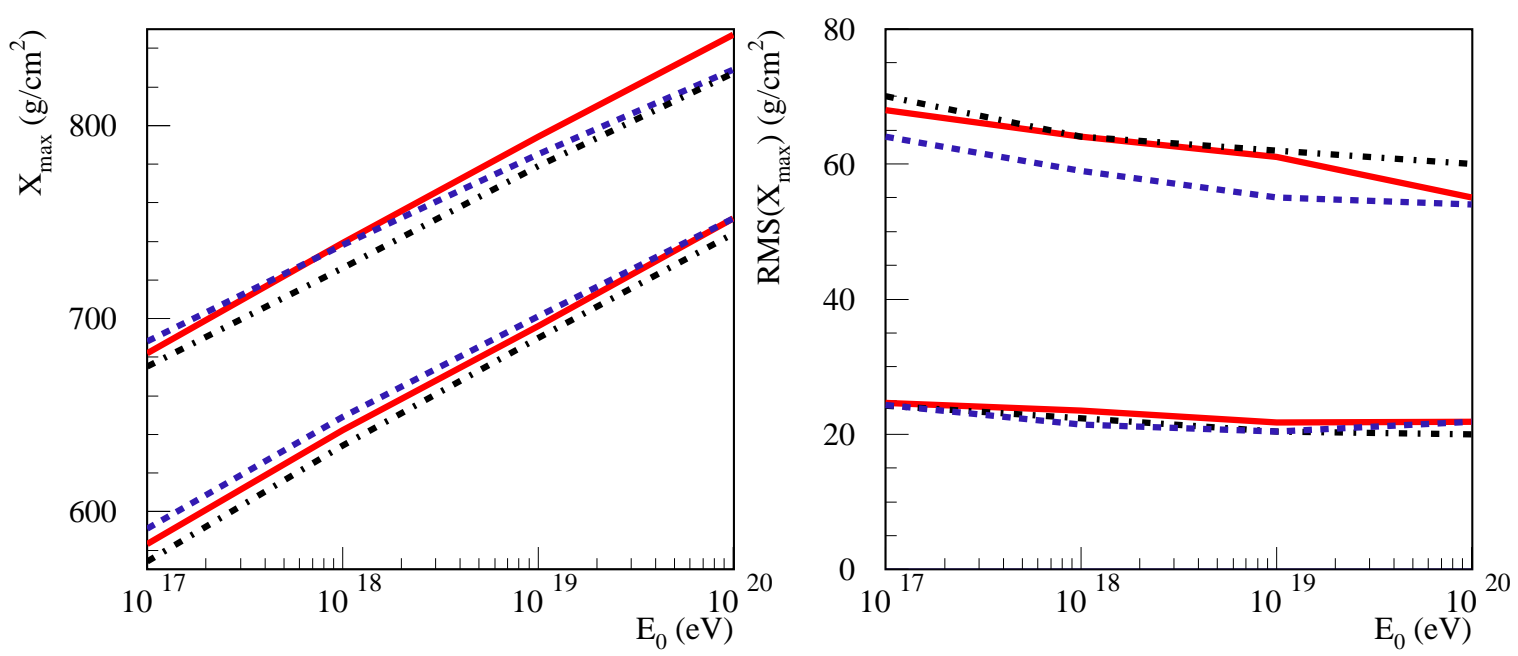

Figure 7. Energy dependence of the shower maximum position $X_{\max }$ (left) and its standard deviation $\mathrm{RMS}\left(X_{\max }\right)$ (right) as calculated using QGSJET-II-04 (solid), QGSJET-II-03 (dashed), and QGSJET (dash-dotted).

One may wonder if LHC results, being obtained mostly for proton-proton collisions, can constrain model predictions for hadron-nucleus and nucleus-nucleus interactions. The conjecture is that the interaction mechanism is the same in all those processes, at least in the framework of the QGSJET-II model: the scattering process is mediated by parton cascades which develop between the projectile and target hadrons (nuclei). It is the larger phase space, primarily, the larger rapidity interval available for such cascades to develop, which defines the energy evolution of the basic observables. Replacing one hadron by another or by a nucleus doesn't change the interaction mechanism, rather changing the initial conditions for those cascades.

However, those initial conditions have a direct impact on the initial stage of such cascades, which is where forward (backward) particle spectra are formed. Thus, the next questions are if the respective description is adequate enough and if an improved treatment may produce a larger $N_{\mu}$. The first question may be answered by comparing model predictions for forward particles spectra with copious accelerator data at fixed target energies - since the relevant mechanisms, like the energy-momentum sharing between multiple parton cascades or production of secondaries by hadronic "remnant" states composed of spectator constituent partons, have the strongest effect there.

To address the second query, let us keep in mind that the only realistic way to significantly enhance EAS muon content is by changing the treatment of pion-air collisions - since the corresponding effect will accumulate over many steps of the nuclear cascade ("hadron generations") in the atmosphere [38]. Further, it has been demonstrated in Ref. [39] that $N_{\mu}$ is rather sensitive to how the leading ("remnant") hadronic states in pion-air scattering are treated, more precisely, if those form multi-particle states or may be represented by a single leading pion. In prin-

mechanism" would produce an excess of muons of relatively low energies $(\sim 1 \mathrm{GeV})$. ciple, this is not surprising since the mechanism influences forward spectra of neutral pions produced and, consequently, the amount of energy going into electromagnetic $(\mathrm{e} / \mathrm{m})$ particles (hence, lost by the hadronic cascade). Meanwhile, as stressed in [39], present CR interaction models tend to predict a somewhat harder $\pi^{0}$ spectra than observed experimentally in $\pi p$-collisions, as illustrated in Fig. 8. The observed model differences at large $x_{\mathrm{F}}$ are

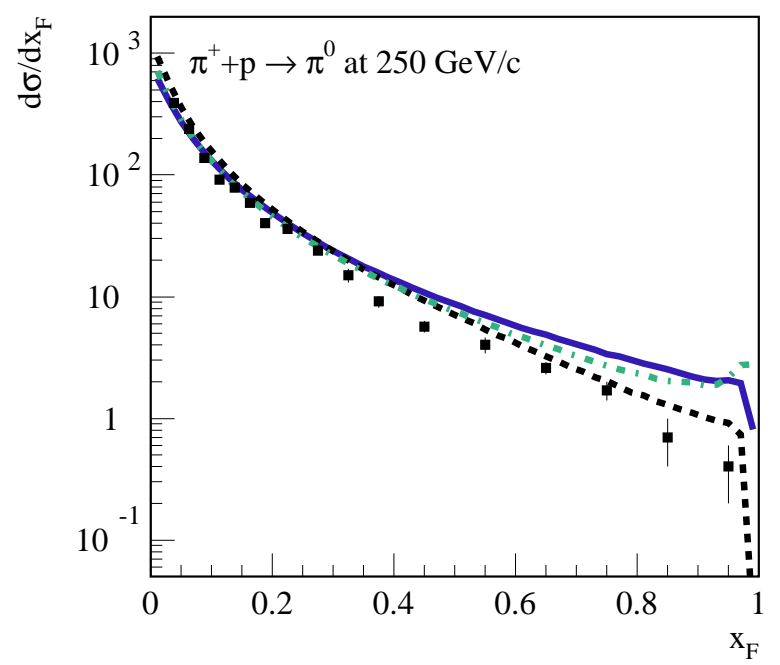

Figure 8. Feynman $x$ spectrum of neutral pions in $\pi^{+} p$ collisions at $250 \mathrm{GeV} / \mathrm{c}$ as calculated with QGSJET-II-03 (solid), QGSJET (dashed), and SIBYLL 2.1 (dash-dotted) compared to experimental data [41] (points).

mostly due to the leading state treatments, e.g. the softer spectrum in QGSJET is because the pion remnant is always represented by a respective low mass excited state.

Thus, we are forced to reconsider the treatment of charge exchange processes in pion-proton and pionnucleus collisions, the importance of the problem being stressed previously in Ref. [40]. Forward neutral hadron production in pion interactions is governed by diagrams of 
the kind depicted in Fig. 9: one considers a slowing down
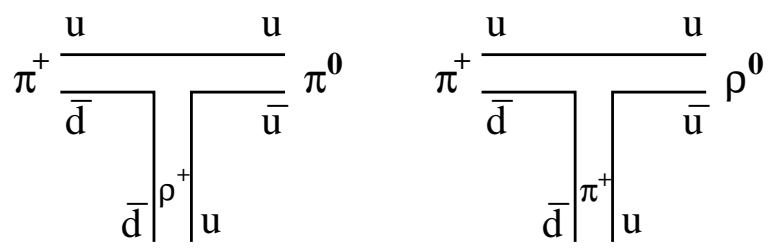

Figure 9. Diagrams for the production of leading neutral meson states in pion collisions: $\rho$-meson exchange (left) and pion exchange (right).

of a valence antiquark (quark) and a creation of a quarkantiquark pair from the vacuum, with the antiquark (quark) from the pair being combined with the remaining valence quark (antiquark) to form a leading hadron. Depending on the parity of the exchanged Reggeon state ( $\bar{d} u$ in Fig. 9), one obtains thus a leading $\pi^{0}\left(\rho^{+}\right.$exchange $)$or $\rho^{0}\left(\pi^{+}\right.$exchange). One can make here a rather radical assumption that the dominant contribution comes from exchanging the Reggeon state of lowest mass, i.e. that pion exchange dominates. In such a case, the obtained very forward spectra of $\rho^{0}$-mesons appear to agree with accelerator data (Fig. $10)$, thus supporting the above-discussed mechanism. One

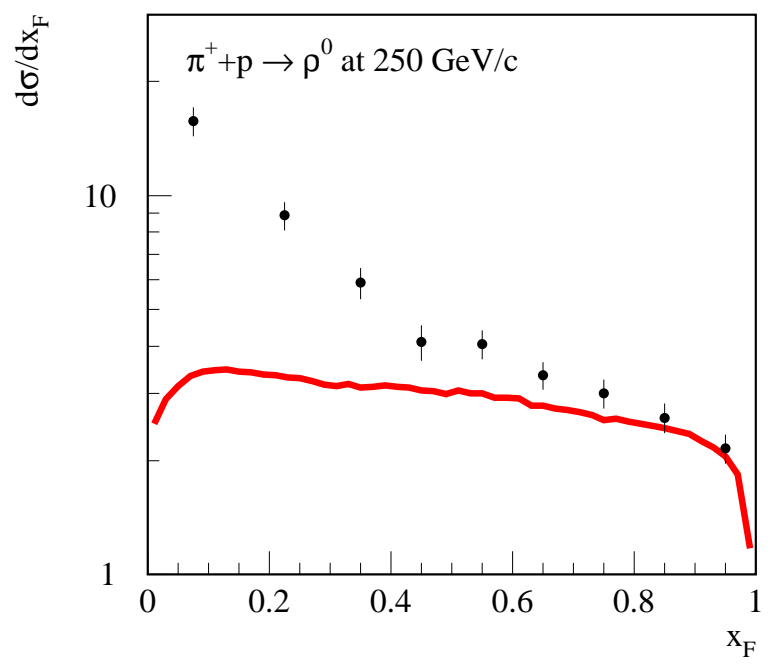

Figure 10. Feynman $x$ spectrum of $\rho^{0}$-mesons in $\pi^{+} p$ collisions at $250 \mathrm{GeV} / \mathrm{c}$ from Ref. [42] (points) compared to the respective "remnant" contribution in QGSJET-II-04 (line) .

obtains also much softer $\pi^{0}$ spectra which come closer to observations, as illustrated in Fig. 11, though experimental uncertainties don't allow one to make definite conclusions here; e.g. the data from Ref. [43] indicate an even softer $\pi^{0}$ spectrum.

As $\rho^{0}$-meson decays into a charged pion pair, the above-discussed modification leads to a higher muon content of air showers. Indeed, the balance between the energy kept in the hadronic cascade and in the e/m one is changed towards the former; one produces a larger number of charged pions per pion-air interaction, with a harder pion spectrum. The resulting changes for EAS muon number are demonstrated in Fig. 12. Clearly, the $N_{\mu}$ enhance-

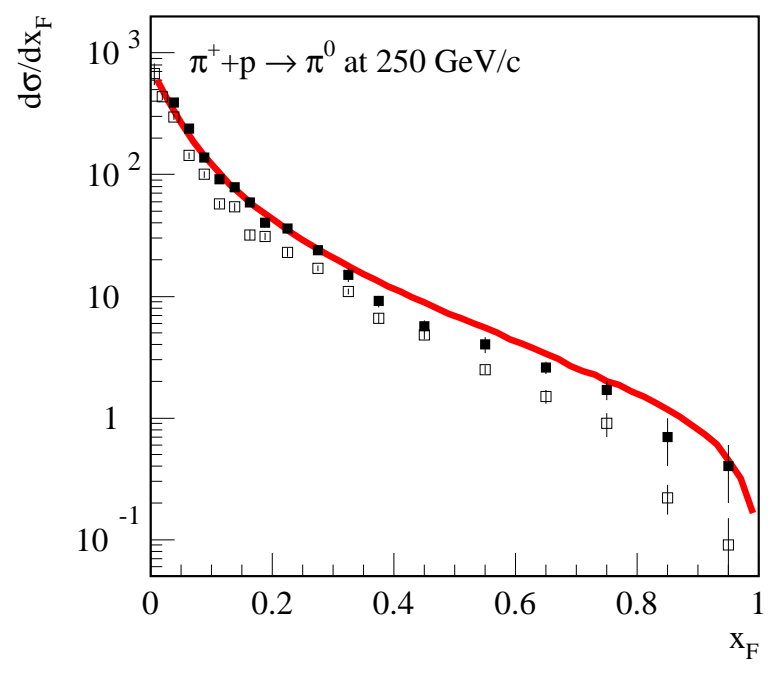

Figure 11. Feynman $x$ spectrum of neutral pions in $\pi^{+} p$ collisions at $250 \mathrm{GeV} / \mathrm{c}$ as calculated with QGSJET-II-04 under the assumtion on pion exchange dominance for charge exchange processes; experimental data are from Ref. [41] (filled squares) and from Ref. [43] (open squares).

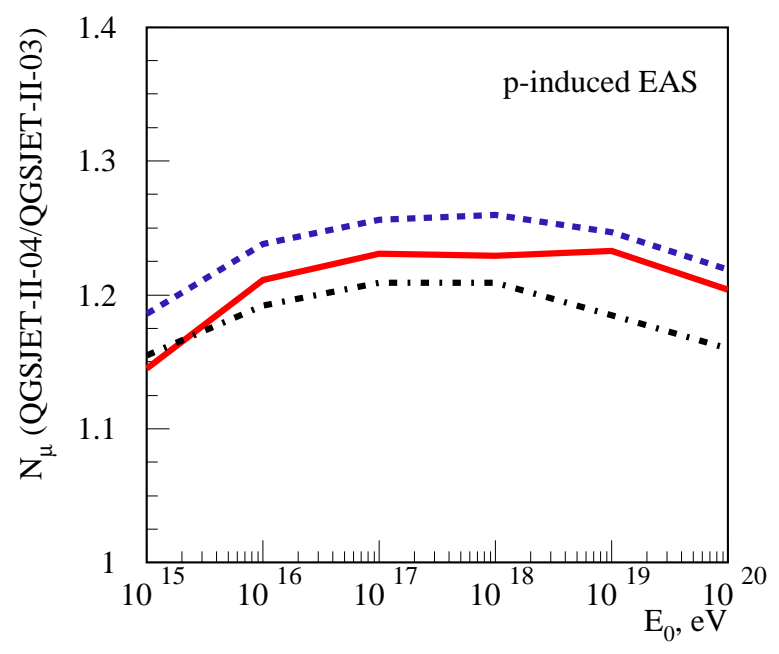

Figure 12. Relative $N_{\mu}$ difference at sea level between QGSJETII-04 and QGSJET-II-03 for proton-induced EAS for different muon energy thresholds: $E_{\mu}>1 \mathrm{GeV}-$ solid, $E_{\mu}>10 \mathrm{GeV}-$ dashed, and $E_{\mu}>100 \mathrm{GeV}$ - dash-dotted.

ment rises with the primary energy due to the larger number of nuclear cascade steps (pion generations), reaching 20-25\% level. However, the effect saturates around $10^{17}$ $\mathrm{eV}$ and weakens at higher energies. The latter is because the bulk of secondary hadron production at high energies comes from parton cascades, with the "remnant" contribution becoming less and less significant with increasing energy. In contrast to the "baryon mechanism", the abovediscussed $N_{\mu}$ enhancement takes place over a wide muon energy range, being maximal for $E_{\mu} \sim 10 \mathrm{GeV}$. Thus, the new model may stay in a somewhat better agreement with the PAO data, though the obtained $N_{\mu}$ enhancement is still insufficient to remove the contradiction, unless a heavy $\mathrm{CR}$ composition is assumed. 

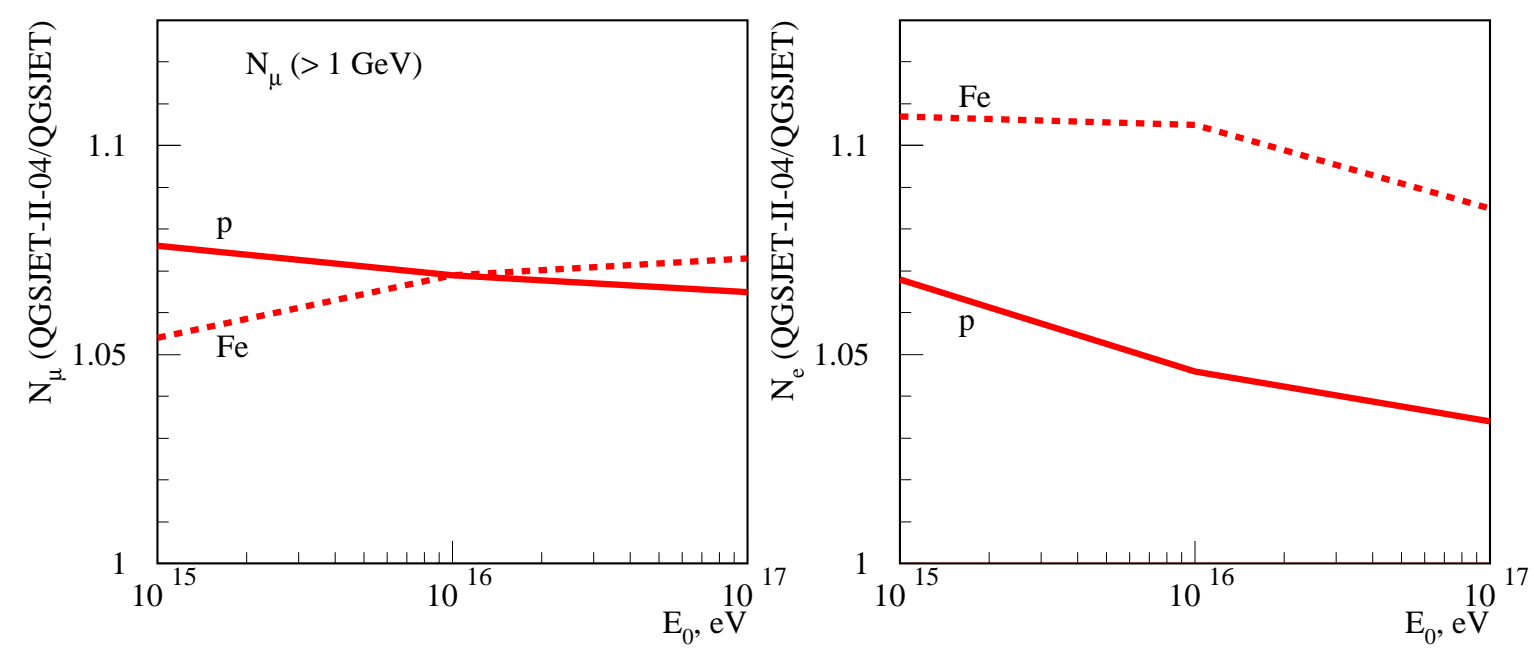

Figure 13. Relative differences between QGSJET-II-04 and QGSJET predictions for muon (left) and electron (right) numbers at sea level for vertical proton- (solid) and iron-induced (dashed) EAS.

Since the obtained enhancement of the muon number is already substantial for primary energies around $10^{15} \mathrm{eV}$, one may question if the new model is consistent with observations of the KASCADE experiment in the "knee" energy range and if it would imply significant changes for CR composition analysis by KASCADE [44, 45], which was based on the QGSJET model. To address these questions, the relative differences between QGSJET-II-04 and QGSJET for the predicted muon $\left(E_{\mu}>1 \mathrm{GeV}\right)$ and electron $\left(e^{ \pm}\right)$numbers for proton- and iron-induced EAS are plotted in Fig. 13. It is easy to see that in the energy range of interest both $N_{e}$ and $N_{\mu}$ are approximately equally enhanced in the new model, particularly for the case of primary protons. Consequently, one can not expect any substantial changes for the derived CR composition which was obtained by unfolding the measured $N_{e}\left(N_{\mu}\right)$ dependence [44]. On the other hand, for $F e$-induced EAS at $10^{15} \mathrm{eV}$, the $N_{e}$ enhancement is about twice the one for the muon number. The corresponding $N_{e}\left(N_{\mu}\right)$ increase is likely to remove the discrepancies between EAS simulations and the KASCADE data in that energy range [44].

\section{Summary}

QGSJET-II offers a phenomenological treatment of hadronic and nuclear collisions at high energies, being developed in the Reggeon Field Theory framework. The soft and semihard parton processes are included in the model within the "semihard Pomeron" approach. Nonlinear interaction effects are treated by means of PomeronPomeron interaction diagrams. By construction, the model is fully consistent with pQCD description in the two key aspects: i) perturbative evolution of parton densities is governed by DGLAP equations; ii) collinear QCD factorization applies for inclusive jet spectra. The explicit treatment of nonlinear processes allows one to treat relevant aspects of the interaction dynamics, in particular, to obtain partial cross sections for various arbitrarily complicated configurations of hadronic and nuclear collisions and to sample the latter using Monte Carlo methods. As an important example, the model predicts a nontrivial dependence of the differential single diffraction cross sections on the diffractive state mass.

The key difference of the present treatment compared to the popular minijet approach is that one doesn't assume the QCD factorization to apply to non-inclusive observables, notably, to the contribution of semihard processes to elastic scattering amplitude. On the contrary, it is demonstrated that nonlinear parton processes explicitly break the factorization for non-inclusive observables and provide non-factorisable screening corrections, in addition to the ones included in nonlinear evolution of parton densities. The latter circumstance appeared to be crucial for obtaining a consistent description of the energy-dependence of total and elastic proton-proton cross sections at very high energies, while staying in agreement with measured parton distributions. As the consequence, the $Q_{0}$-cutoff is a technical energy-independent parameter of the scheme, used to separate short- and long-distance physics.

On the other hand, the treatment doesn't take into account "hard" Pomeron-Pomeron coupling corresponding to nonlinear parton processes in the perturbative domain, thus missing the respective screening effects. This poses limitations to model applications for a treatment of central nucleus-nucleus collisions at very high energies.

The latest model version, QGSJET-II-04, comprises three important updates: i) treatment of all significant enhanced diagram contributions to the underlying dynamics, including ones of Pomeron loops; ii) re-calibration of the model with new LHC data; iii) improved treatment of charge exchange processes in pion-proton and pionnucleus collisions.

Compared to the previous model version (QGSJET-II03), QGSJET-II-04 predicts a somewhat higher elongation rate for extensive air shower maximum position $X_{\max }$, the respective difference reaching $20 \mathrm{~g} / \mathrm{cm}^{2}$ at the highest CR energies. Also slightly larger $X_{\max }$ fluctuations (by some 5 $\mathrm{g} / \mathrm{cm}^{2}$ ) are obtained for proton-induced EAS, both effects 
being driven by the model calibration to TOTEM data on total and elastic proton-proton cross sections.

For the shower muon content, one obtained $\sim 20 \%$ enhancement, the effect being mostly due to the modified treatment of charge exchange processes in pion collisions. Importantly, this enhancement is approximately muon energy-independent in the range $E_{\mu} \simeq 1-100 \mathrm{GeV}$, which thus offers a possibility to soften the disagreement between the respective PAO results and EAS simulations. On the other hand, in the "knee" energy range, the obtained $N_{e}\left(N_{\mu}\right)$ dependence is similar to the one for the old QGSJET model. Hence, no significant changes for the respective CR composition analysis by the KASCADE collaboration should be expected.

\section{Acknowledgments}

This work was supported by the program Romforskning of Norsk Forsknigsradet.

\section{References}

[1] S. Ostapchenko, Phys. Rev. D74, 014026 (2006).

[2] S. Ostapchenko, Nucl. Phys. Proc. Suppl. 151, 143 (2006).

[3] S. Ostapchenko, Phys. Rev. D83, 014018 (2011).

[4] L. Gribov, E. Levin and M. Ryskin, Phys. Rep. 100, 1 (1983).

[5] T. K. Gaisser and F. Halzen, Phys. Rev. Lett. 54, 1754 (1985).

[6] L. Durand and P. Hong, Phys. Rev. Lett. 58, 303 (1987).

[7] F. W. Bopp et al., Phys. Rev. D49, 3236 (1994).

[8] A. H. Mueller and J. Qui, Nucl. Phys. B268, 427 (1986).

[9] S. Li and X.-N. Wang, Phys. Lett. B527, 85 (2002).

[10] N. N. Kalmykov, S. S. Ostapchenko and A. I. Pavlov, Bull. Russ. Acad. Sci. Phys. 58, 1966 (1994) [Izv. RAN Ser. Fiz. 58, 21 (1994)]; Nucl. Phys. Proc. Suppl. 52B, 17 (1997).

[11] H.J. Drescher et al., Phys. Rep. 350, 93 (2001).

[12] V. N. Gribov, Sov. Phys. JETP 26, 414 (1968).

[13] H.J. Drescher et al., J. Phys. G25, L91 (1999); S. Ostapchenko et al., J. Phys. G28, 2597 (2002).

[14] V. A. Abramovskii, V. N. Gribov and O. V. Kancheli, Sov. J. Nucl. Phys. 18, 308 (1974).

[15] O. V. Kancheli, JETP Lett. 18, 274 (1973); J. L. Cardi, Nucl. Phys. B75, 413 (1974); A. B. Kaidalov, L. A. Ponomarev and K. A. Ter-Martirosyan, Sov. J. Nucl. Phys. 44, 468 (1986).

[16] S. Ostapchenko, Phys. Lett. B636, 40 (2006).
[17] S. Ostapchenko, Phys. Rev. D77, 034009 (2008).

[18] S. Ostapchenko, Phys. Rev. D81, 114028 (2010).

[19] E.-J. Ahn et al., Phys. Rev. D80, 094003 (2009).

[20] S. Ostapchenko, Phys. Lett. B703,588 (2011).

[21] G. Aad et al. (ATLAS Collaboration), Eur. Phys. J. C72, 1926 (2012).

[22] G. Anelli et al. (TOTEM Collaboration), JINST 3, S08007 (2008).

[23] S. Ostapchenko, AIP Conf. Proc. 928, 118 (2007).

[24] T. C. Rogers and M. Strikman, Phys. Rev. D81, 016013 (2010).

[25] M. Braun, Sov. J. Nucl. Phys. 52, 164 (1990); V. A. Abramovskii and G. G. Leptoukh, ibid. 55, 903 (1992); M. Hladik et al., Phys. Rev. Lett. 86, 3506 (2001).

[26] S. Ostapchenko, T. Pierog and K. Werner, Nucl. Phys. Proc. Suppl. 122, 400 (2003).

[27] S. Ostapchenko, Nucl. Phys. Proc. Suppl. 196, 90 (2009).

[28] G. Antchev et al. (TOTEM Collaboration), Europhys. Lett. 96, 21002 (2011).

[29] S. Ostapchenko, Prog. Theor. Phys. Suppl. 193, 204 (2012).

[30] K. Nakamura et al. (Particle Data Group), J. Phys. G37, 075021 (2010).

[31] N.N. Kalmykov and S.S. Ostapchenko, Sov. J. Nucl. Phys. 50, 315 (1989); Phys. Atom. Nucl. 56, 346 (1993).

[32] J. Engel et al., Phys. Rev. D46, 5013 (1992).

[33] R. Aloisio et al., Phys. Rev. D77, 025007 (2008).

[34] J. Allen for the Pierre Auger Collaboration), Proc. 32nd ICRC (2011), arXiv:1107.4809 [astro-ph.HE].

[35] D. d'Enterria et al., Astropart. Phys. 35, 98 (2011).

[36] P. K. F. Grieder, Proc. of the 13th Int. Conf. on Cosmic Rays, Denver (Colorado), USA, v. 4, p. 2467.

[37] T. Pierog and K. Werner, Phys. Rev. Lett. 101, 171101 (2008).

[38] S. Ostapchenko, Czech J. Phys. 56, A149 (2006).

[39] H. J. Drescher, Phys. Rev. D77, 056003 (2007).

[40] A. D. Erlykin, Astropart. Phys. 27, 521 (2007).

[41] M. Adamus et al. (NA22 Collaboration), Z. Phys. C35, 7 (1987).

[42] N. M. Agababyan et al. (EHS-NA22 Collaboration), Z. Phys. C46, 387 (1990).

[43] M. R. Ataian et al. (EHS-NA22 Collaboration), Z. Phys. C54, 247 (1992).

[44] T. Antoni et al. (KASCADE Collaboration), Astopart. Phys. 24, 1 (2005).

[45] W. D. Apel et al. (KASCADE-Grande Collaboration), Astopart. Phys. 31, 86 (2009). 